\title{
Quantum simulation of artificial Abelian gauge field using nitrogen-vacancy center ensembles coupled to superconducting resonators
}

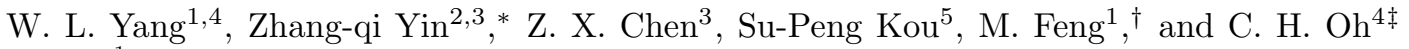 \\ ${ }^{1}$ State Key Laboratory of Magnetic Resonance and Atomic and Molecular Physics, \\ Wuhan Institute of Physics and Mathematics, Chinese Academy of Sciences, \\ and Wuhan National Laboratory for Optoelectronics, Wuhan 430071, China \\ ${ }^{2}$ Key Laboratory of Quantum Information, University of Science and Technology of China, \\ Chinese Academy of Sciences, Hefei 230026, China \\ ${ }^{3}$ Center for Quantum Information, IIIS, Tsinghua University, Beijing, China \\ ${ }^{4}$ Centre for Quantum Technologies, National University of Singapore, Singapore 117543, Singapore and \\ ${ }^{5}$ Department of Physics, Beijing Normal University, Beijing 100875, China
}

\begin{abstract}
We propose a potentially practical scheme to simulate artificial Abelian gauge field for polaritons using a hybrid quantum system consisting of nitrogen-vacancy center ensembles (NVEs) and superconducting transmission line resonators (TLR). In our case, the collective excitations of NVEs play the role of bosonic particles, and our multiport device tends to circulate polaritons in a behavior like a charged particle in an external magnetic field. We discuss the possibility of identifying signatures of the Hofstadter "butterfly" in the optical spectra of the resonators, and analyze the ground state crossover for different gauge fields. Our work opens new perspectives in quantum simulation of condensed matter and many-body physics using hybrid spin-ensemble circuit quantum electrodynamics system. The experimental feasibility and challenge are justified using currently available technology.
\end{abstract}

PACS numbers: 03.67.Bg, 76.30.Mi, 42.50.Pq

Gauge field theory [1], which was developed for describing the subatomic interaction, has deepened our understanding of a wide range of physical phenomena: quantum magnetoresistance oscillations, superconducting vortices, quantum Hall effects (QHE), and Hofstadter butterfly (HB) 22] etc. However, these phenomena associated with the gauge field are very difficult to be directly observed in ordinary solid-state systems due to the requirement of extremely high field. Recently, following the idea of quantum simulation [3], several theoretical schemes have been proposed to generate artificial gauge field in atomic, molecular, and optical systems. The first route is taken in cold atomic systems. The gauge field can be generated by rotating the trap or lattice [4, 5$]$, by introducing appropriate phase factors for hopping amplitudes through combining Raman-laser-assisted tunneling and lattice acceleration methods [6 -10], or by tailoring orthogonal laser-beam coupled to the atomic degenerate internal states [11, 12], or by simply using the ordinary tunneling in an optical lattice [13]. The second route is based on cavity quantum electrodynamics (QED) systems, where the gauge field can also appear through manipulating the phase factors of the hopping term, in circuit QED cavity arrays [14], in confined ions in microtrap array [15], in coupled resonator optical waveguides [16], and in solid-state photonic structures [17]. In addition, the fractional quantum Hall effect (FQHE) can also be simulated in optical-cavity arrays by trapping three-level

\footnotetext{
*Electronic address: yinzhangqi@gmail.com

$\dagger$ Electronic address: mangfeng@wipm.ac.cn

‡Electronic address: phyohch@nus.edu.sg
}

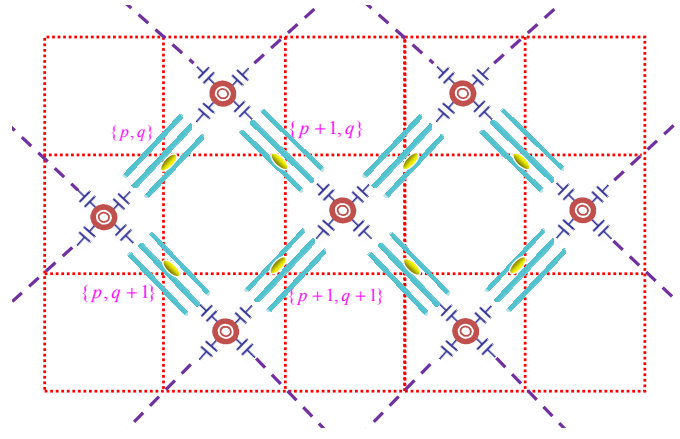

FIG. 1: (Color online) Schematic circuit for the resonator array, where the NVEs are magnetically coupled to the quantized field of the TLR, and the circles denote the central coupler. Microwave photons can tunnel between adjacent resonators via capacitive coupling. The lattice sites are represented by $a(p \hat{x}+q \hat{y})$ with $a$ the spacing of the lattices, where the dotted grid denote the 'vertical' and 'horizontal' direction.

atoms and using elaborated laser driving [18]. However, due to the required rigorous conditions, up to now only few pioneering experiments have successfully mimicked the effect of a light-induced artificial magnetic field in an optical lattice 20] and a synthetic electric field BoseEinstein condensates [21] by using a Raman-assisted tunneling method [9], and an effective time-dependent vector potential, respectively.

In this paper we propose an alternative theoretical approach for quantum simulating an effective gauge potential in a hybrid solid-state system. We consider NVEs [22 28] which are confined in two-dimensional $(2 D)$ square lattices of superconducting microwave TLR [29]. The NVEs are driven by series of microwave sources 


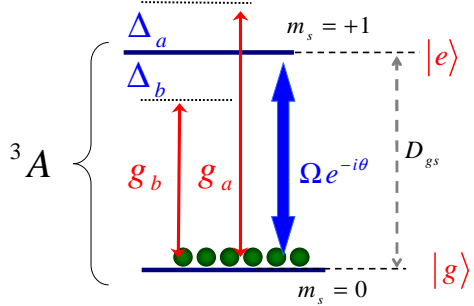

FIG. 2: (Color online) Level structure of a NVE, where the electronic ground state is an electron spin triplet state $(\mathrm{S}=1)$, and $D_{g s} / 2 \pi=2.87 \mathrm{GHz}$ is the zero-field splitting between the $m_{s}=0$ sublevel and the $m_{s}= \pm 1$ sublevels in the absence of external magnetic field. The degeneracy between states $m_{s}= \pm 1$ is lifted by applying a static magnetic field with $40 m T$ parallel to the chip and along the [111] crystalline axis within a few degrees. With this orientation, the four equivalent NV center crystalline orientations $\langle 111\rangle$ all make approximately the same angle $55^{\circ}$ with the magnetic field so that their resonance frequencies are approximately equal. The transition between $|g\rangle$ and $|e\rangle$ with Larmor frequency $\omega_{e}$ is coupled to the two-mode TLR with the coupling rates $g_{a}$ $\left(g_{b}\right)$ and detunings $\Delta_{a}\left(\Delta_{b}\right)$, and is also driven by a classical field with the Rabi frequency $\Omega$ and the related phase $\theta$.

with different phases at different sites. We carefully tune the frequencies of the cavity modes, the driving strength and the phase of microwave sources, in order to induce the non-zero tunneling phases around a closed loop in real space and to generate a non-vanishing artificial gauge field experienced by the orbital motion of the polaritons in NVEs. Our approach utilizes a space-dependent coupling between internal ground states of the NVEs, which yields crucial phases to create considerable synthetic gauge fields. This intriguing behavior is indeed the analogue of the motion of charged particle in an real magnetic field.

The main merits of the present system include the in situ tunability of the parameters of the circuit elements, individual addressing, the peculiar characteristics of the NVE [30] (e.g., long coherence time at roomtemperature), and the scalability of cavity resonator arrays [14, 31, 32]. Very recently, D. Underwood et al experimentally demonstrated 25 arrays of twelve capacitively coupled TLRs and accessed the feasibility of quantum simulation in cavity QED systems [33]. It implies that the polariton-polariton interaction between distant NVEs can be effectively tuned in a controllable way, which renders our scheme to be more practical. By combining the related spectroscopic circuit QED technique for readout of the quantum states of individual constituent elements, we could probe the properties of the system by independently detecting the correlation between distant sites or output fields of the TLRs, which provides the feasibility of observing the strong gauge field effects, such as HB spectrum, in a realistic hybrid solidstate system.

As illustrated in Fig. 1, the system we study is a $2 D$ square lattice, where the basic unit consists of a NVE confined in a microwave-driven two-mode TLR with the length $L$, the inductance $F_{t}$ and the capacitance $C_{t}$, a narrow center conductor and two nearby lateral ground planes. Each NVE has the average NV center density $1 \times 10^{15} \mathrm{~cm}^{-3}$ [34], where each NV center is negatively charged with two unpaired electrons located at the vacancy, which can be modeled as a two-level system in the ground-state subspaces as shown in Fig. 2. For clarity of description, we adopt following denotations: $\left|{ }^{3} A, m_{s}=0\right\rangle=|g\rangle$, and $\left|{ }^{3} A, m_{s}=+1\right\rangle=|e\rangle$ (we distinguish the degenerate sublevels $\left.\left.\right|^{3} A, m_{s}= \pm 1\right\rangle$ by appropriate external magnetic field or polarized irradiation). In our system, microwave-photons can tunnel between adjacent TLRs via capacitive coupling by connecting TLRs via a central coupler, which serves as individual tunable quantum transducers to transfer photonic states between the resonators.

The overall system is governed by the Hamiltonian $H_{t o t}=\sum_{p, q}\left(H_{C}^{(p, q)}+H_{J C}^{(p, q)}+H_{N V}^{(p, q)}+H_{T}^{(p, q)}\right)$, where $H_{C}^{(p, q)}=\sum_{c=a, b} \hbar \omega_{c} c_{p, q}^{\dagger} c_{p, q}$ is for the $(p, q)$-th TLR's mode with $c_{p, q}^{\dagger}\left(c_{p, q}\right)$ the creation (annihilation) operators of the full-wave mode of the resonator and $\omega_{c}=$ $2 \pi /\left(\sqrt{F_{t} C_{t}}\right)$ the corresponding eigenfrequency. The collectively magnetic coupling between NVE and all the microwave modes can be modeled as

$H_{J C}^{(p, q)}=\sum_{p, q} \sum_{c=a, b} \hbar\left(g_{p, q}^{c} S_{p, q}^{+} c_{p, q}+\Omega_{p, q} e^{i\left(\omega_{l} t+\theta_{p, q}\right)} S_{p, q}^{+}+H . C.\right)$,

where $g_{p, q}^{c}$ is the single NV center's vacuum Rabi frequency in the $(p, q)$-th resonator for mode $c$, and $\omega_{l}$ is the frequency of the external driving laser with the Rabi frequency $\Omega_{p, q}$ and the phase $\theta_{p, q}$. The collective transition operators of NVE are defined as $S_{p, q}^{+}=\sum_{j=1}^{N_{p, q}}|e\rangle_{p, q p, q}^{j}\langle g|$ and $S_{p, q}^{-}=\sum_{j=1}^{N_{p, q}}|g\rangle_{p, q p, q}^{j}\langle e|$ with $N_{p, q}$ the number of the NV centers in a NVE inside the $(p, q)$-th resonator.

The Hamiltonian of a NVE reads $H_{N V}^{(p, q)}=\frac{\hbar}{2} \omega_{e} S_{p, q}^{z}$ with $\omega_{e}$ the energy-level spacing of the states $|e\rangle$ and $|g\rangle$. All the spins in NVE interact symmetrically with a single mode of electromagnetic field by magnetic-dipole coupling because the mode wavelength is larger than the spatial dimension of the NVE if the spin ensemble is placed near the TLR's field antinode. We suppose that the mode $a_{p, q}$ couples with the vertical adjacent sites, and $b_{p, q}$ couples with the horizontal adjacent sites. So the tunneling between adjacent sites can be governed by the Hamiltonian $H_{T}^{(p, q)}=-\hbar\left(T_{a} a_{p, q+1}^{\dagger} a_{p, q}+T_{a} a_{p, q}^{\dagger} a_{p, q+1}+\right.$ $\left.T_{b} b_{p+1, q} b_{p, q}^{\dagger}+T_{b} b_{p, q} b_{p+1, q}^{\dagger}\right)$. Here the intercavity photonic tunneling rates $T_{a}\left(T_{b}\right)$ are tunable experimental parameters due to the flexibility of the central coupler. The central coupler may be conceived as a current-biased Josephson junction phase qubit [35], or Josephson ring circuit [14], or a capacitive coupling element 36], or an active non-reciprocal devices as proposed in [37].

Under the strong driving case $\Omega_{p, q} \gg T_{c}, \sqrt{N_{p, q}} g_{p, q}^{c}$, 
we define the new energy levels as $|+\rangle_{p, q}=\left(e^{i \theta_{p, q}}|e\rangle_{p, q}+\right.$ $\left.|g\rangle_{p, q}\right) / \sqrt{2}$ and $|-\rangle_{p, q}=\left(-e^{i \theta_{p, q}}|e\rangle_{p, q}+|g\rangle_{p, q}\right) / \sqrt{2}$. Introducing $\Delta_{c}=\omega_{c}-\omega_{e}$ and $\delta_{p, q}^{c}=\Delta_{c}-\Omega_{p, q}$, and supposing $\left|\delta_{p, q}^{c}\right| \ll \Omega_{p, q}$, we have the effective Hamiltonian of the mode $a$ in lattice site $(p, q)$ under the new basis as $H_{p, q}^{a}=\delta_{p, q}^{a} a_{p, q}^{\dagger} a_{p, q}+\left(\frac{1}{2} g_{p, q}^{a} e^{-i \theta_{p, q}} a_{p, q} \tilde{S}_{p, q}^{+}+\right.$H.C. $)$, with $\tilde{S}_{p, q}^{+}=\sum_{j=1}^{N_{p, q}}|+\rangle_{p, q p, q}^{j}\langle-|$ (see Sec.I in supplementary material). On the other hand, the effective Hamiltonian describing the mode $b$ is $H_{p, q}^{b}=\delta_{p, q}^{b} b_{p, q}^{\dagger} b_{p, q}-$ $\left(\frac{1}{2} g_{p, q}^{b} e^{-i \theta_{p, q}} b_{p, q}^{\dagger} \tilde{S}_{p, q}^{+}+\right.$H.C. $)$.

For simplicity, we suppose $\delta_{p, q}^{c}=\delta, T_{c}=T$, and $g_{p, q}^{c}=g$ for all $p, q, c$. In the limit of $\delta \gg T, g$, we can adiabatically eliminate the photonic modes and get the effective Hamiltonian between NVEs (see Sec.II in supplementary material),

$$
\begin{aligned}
H_{\mathrm{eff}}= & -J^{\prime}\left(\sum_{p, q} e^{i\left(\theta_{p, q}-\theta_{p+1, q}\right)} \tilde{S}_{p, q}^{+} \tilde{S}_{p+1, q}\right. \\
& \left.+e^{i\left(\theta_{p, q+1}-\theta_{p, q}\right)} \tilde{S}_{p, q}^{+} \tilde{S}_{p, q+1}+\text { H.C. }\right)
\end{aligned}
$$

where $J^{\prime}=T(g / 2 \delta)^{2}$.

Using the Holstein-Primakoff transformation $\tilde{S}^{+}=B_{p, q}^{\dagger} \sqrt{N_{p, q}-B_{p, q}^{\dagger} B_{p, q}} \simeq \sqrt{N_{p, q}} B_{p, q}^{\dagger}$, $\tilde{S}_{p, q}^{-}=B_{p, q} \sqrt{N_{p, q}-B_{p, q}^{\dagger} B_{p, q}} \simeq \sqrt{N_{p, q}} B_{p, q}$, and $\tilde{S}_{p, q}^{z}=\left(B_{p, q}^{\dagger} B_{p, q}-N_{p, q} / 2\right)$ [38], where the operator $B_{p, q}^{\dagger}\left(B_{p, q}\right)$ fulfills the bosonic commutation relation $\left[B_{p, q}, B_{p^{\prime}, q^{\prime}}^{\dagger}\right] \simeq \delta_{p q, p^{\prime} q^{\prime}}$ in the case of low number of NVE excitations, we can map the collective raising (lowering) operators $\tilde{S}_{p, q}^{ \pm}$of the $(p, q)$-th NVE into the bosonic operators $B_{p, q}^{\dagger}$ and $B_{p, q}$. These transformations change the Hamiltonian $H_{\text {eff }}$ into a concise form as

$$
\begin{aligned}
H_{B}= & -J\left(\sum_{p, q} e^{i\left(\theta_{p, q}-\theta_{p+1, q}\right)} B_{p, q}^{\dagger} B_{p+1, q}\right. \\
& \left.+e^{i\left(\theta_{p, q+1}-\theta_{p, q}\right)} B_{p, q}^{\dagger} B_{p, q+1}+\text { H.C. }\right),
\end{aligned}
$$

where $J=N_{p, q} J^{\prime}=T\left(g_{e f f} / 2 \delta\right)^{2}$ and the collective coupling rates $g_{\text {eff }}$ between NVE and TLR in each lattice site are supposed to be equal in our case.

Because of the spatial variation of the tunneling phase, the wave function of a polariton from one lattice site to another acquires a nontrivial phase, which can be interpreted as an effective Aharonov-Bohm phase. With a suitable tuning of the tunneling phases between pairs of neighboring NVEs, the polariton turns out to experience a non-trivial artificial gauge potential $\vec{A}$, which can be identified by

$$
\theta_{p, q}=\frac{e}{\hbar} \int_{0, q}^{p, q} \vec{A} \cdot d \vec{L}=\frac{e}{\hbar} \int_{p, q}^{p, 0} \vec{A} \cdot d \vec{L},
$$

where the integral is performed along the segment connecting the TLRs, and $e$ is the effective charge. With the choice of the symmetric gauge, we set $\theta_{p, q}=-\pi p q \alpha$,
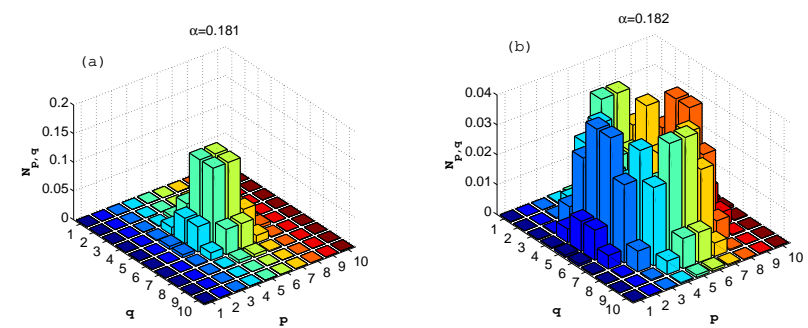

FIG. 3: (Color online) For $10 \times 10$ lattice, the spatial distribution of absolute square of the polariton $B_{p, q}$ wave function in the ground state of Hamiltonian (3) with open boundary conditions, under different values of $\alpha$ : (a) $\alpha=0.181$. (b) $\alpha=0.182$.

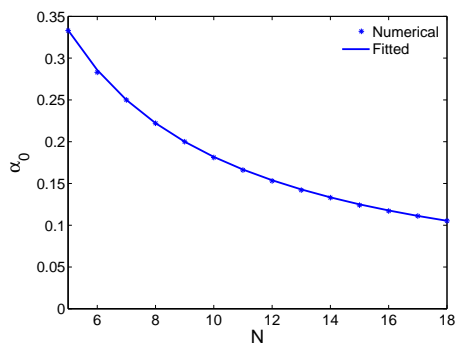

FIG. 4: (Color online) The relation between the critical value of $\alpha_{0}$ and the system size $N$.

where $\alpha=\Phi / \Phi_{0}$ with $\Phi$ the magnetic flux through a unit cell, and $\Phi_{0}=h / e$ the flux quantum. So a uniform artificial gauge field will emerge during the polaritonic dynamics. The sum of the tunneling phases along a closed loop surrounding the plaquette is $2\left(\theta_{p+1, q}+\right.$ $\left.\theta_{p, q+1}-\theta_{p, q}-\theta_{p+1, q+1}\right)=2 \pi \alpha$, which is actually the flux quanta per plaquette, namely, the strength of the artificial gauge field. Note that the phase errors of the local microwave source only cause the local gauge field variance. The total flux remains unchanged.

Next we focus on the observable consequences of a uniform magnetic field in the present system. For simplicity, we consider the case of non-interacting polaritons in finite-size $2 D$ square lattice under the tight-binding limit and the single-polariton subspace. As shown in Fig. 3, we plot the spatial distribution of polaritons $B_{p, q}$ in the ground state of the $10 \times 10$ lattice under different values of $\alpha$. When $\alpha=0$, the Hamiltonian in Eq. (3) can be reduced to that for a free particle in an infinitely deep square potential well and the form of ground state takes the Sine functions. When $\alpha$ increases from 0 to $\alpha_{0} \simeq 0.181$, we find that the polaritons concentrate at the central region which implies the wave-function of the ground state gradually change from Sine function into that of the 0-th Landau level with zero angular momentum $m$, and the radius of the 0th Landau level decreases as the gauge field increases. This transition which is denoted by $\alpha$ is really from 0 -th Landau level of $m=0$ to 0 -th Landau level of $m>0$. However the distribution 
changes dramatically once the value of $\alpha$ exceeds a critical value $\alpha_{0}$, where the 0 -th Landau level with $m>0$ momentum becomes ground state. The physical mechanism behind this intriguing phenomena is the competition between the gauge field and the size effect. As shown in Fig. 4 , we find that the crossing point $\alpha_{0}$ can be well fitted by the equation $\alpha_{0}=2 /(N+1)$ for the $N \times N$ systems with $N>4$ (see Sec. III in supplementary material). If we further increase $\alpha$, many level crossing points would appear. We find that all the crossing points are near certain rational points $\alpha=p / q$, at which the wave-functions show regular oscillations [2]. After Fourier transformation, one can see that such regular oscillations come from the coherent interference between the peculiar points in momentum space (see Sec. III in supplementary material). It is very interesting that the results from the model with the periodic condition in thermodynamic limit can be observed in this small and open boundary system. In order to measure the level crossing, we can couple the NVEs to superconducting qubits, and transfer the state of NVEs to the nearby qubits. Then we can measure the ground state population distribution of polaritons by measuring the states of superconducting qubits.

To investigate the possibility of observing the fractal band structure of $\mathrm{HB}$, we diagonalize the Hamiltonian $H_{B}$ in the single-polariton subspace for several small lattice sizes. As shown in Fig. 5a, the HB structure can be observed in the size of $5 \times 5$. It becomes clearer in the whole transmission spectra with the growth of the size. Experimentally, transmission and reflection measurements have been by far performed routinely in smallscale circuit QED systems with one or two resonators [29, 40]. We can characterize our hybrid circuits by spectroscopic measurements, and a feasible method is to measure the resonator transmission through a network analyzer. In realistic experiments, the transmission spectrum of these superconducting resonators will show a series of Lorentzian peaks, whose central frequencies correspond to the eigenfrequencies of the Hamiltonian $H_{\text {eff }}$ in Eq. (3). Alternatively, using homodyne detection followed by sampling and averaging after amplification, we may reveal the coupled resonator-spin dynamics by measuring the amplitude of the exponentially damped microwave signal that leaks out of the resonators after its interaction with the spin ensembles [41]. So far it is still a great challenge to observe such a fascinating structure in ordinary solid-state system, where the major challenge comes from the extremely high magnetic field required in realistic experiments [42]. In this sense, our proposal opens an entirely new arena to investigate condensed matter and many-body system with light. Meanwhile, the experimental progress, especially in large-scale circuit QED [31, 32], has raised the possibility of observing the HB spectrum directly in realistic experiments [43].

Finally, we survey the relevant experimental parameters. First, the TLR cavity with an inductance $F_{t}=45.6$ $\mathrm{nH}$ and capacitance $C_{t}=2 \mathrm{pF}$ leads to a full wave frequency $\omega_{0} / 2 \pi=D_{g s}+\Delta_{c}=3.31 \mathrm{GHz}$ with $\Delta_{c} / 2 \pi=440$
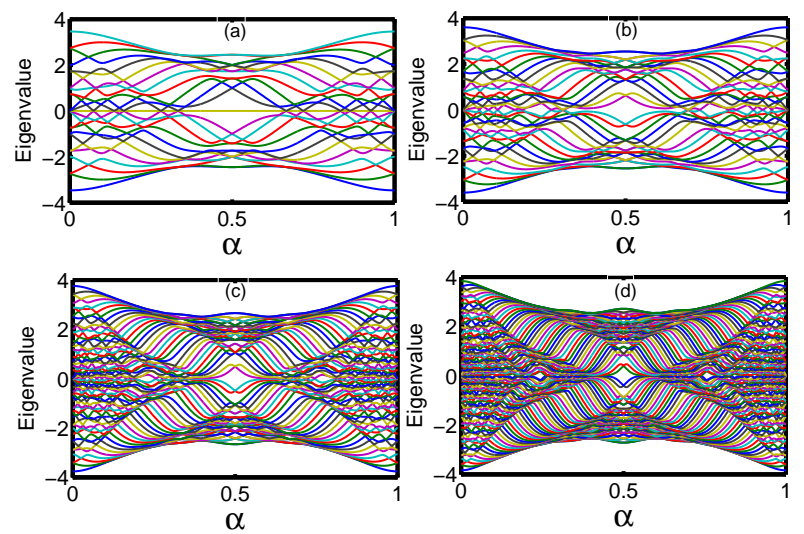

FIG. 5: (Color online) The energy spectrum for (a) $5 \times 5$ lattice; (b) $6 \times 6$ lattice; (c) $8 \times 8$ lattice; (d) $10 \times 10$ lattice, where energy is in units of tunneling strength $J$. The vertical axis is the eigenenergy of the system, and the horizontal axis indicates magnetic flux through a unit cell.

$\mathrm{MHz}$. Second, our scheme requires the large-detuning condition, namely, the detuning $\Delta_{c} \gg g_{\text {eff }}$. Third, the classical field should be tuned to $\Omega / 2 \pi=400 \mathrm{MHz}$ $\gg g_{e f f}$, which makes the energy shift induced by the cavity mode negligible [47]. So if we take the values of the parameters $g_{\text {eff }} / 2 \pi=8 \mathrm{MHz}, T / 2 \pi=4 \mathrm{MHz}$, and $\delta / 2 \pi=40 \mathrm{MHz}$, yielding $J / 2 \pi=T g_{\text {eff }}^{2} / 4 \delta^{2}=0.04$ $\mathrm{MHz}$, our scheme is feasible. This could be confirmed by recent experimental demonstration of coherent coupling of a NVE and a superconducting flux qubit 48. as well as the experimental advances in excellent quantum control with strong magnetic coupling $(\sim 2 \pi \times 10$ $\mathrm{MHz}$ ) between TLR and NVE and the cavity linewidth of $\kappa / 2 \pi \sim \mathrm{kHz}[22-25]$. On the other hand, the electron spin relaxation time $T_{1}$ of NV centers ranges from $6 \mathrm{~ms}$ at room temperature 49 to $28 \sim 265$ s at low temperature [50]. In addition, the dephasing time $T_{2}>600 \mu \mathrm{s}$ for NVE with natural abundance of ${ }^{13} C$ has been reported [51]. A later experimental progress [52] with isotopically pure diamond sample has demonstrated a longer dephasing time to be $T_{2}=1.8 \mathrm{~ms}$. Therefore, the parameter $J$ is higher by nearly two orders of magnitude than the dissipation rates of the NVE, which makes reliable quantum simulation feasible.

Compared with previous cavity QED protocols [18, 53], our scheme requires only one driving source at each site, and the effective couplings between neighboring spins are much larger than in Ref. [18] because the excited states are not required to be adiabatically eliminated in our case. However, so far we have ignored the detrimental influence from the nuclear spin, such as ${ }^{13} C$ defects, in the NVE, which could be alleviated by isotopically purified ${ }^{12} \mathrm{C}$ diamond through the purification technique [26, 52]. Another decoherence source is the dipole-interaction between the redundant Nitrogen spins and the NV centers, which could be reduced by improving the nitrogen to $\mathrm{N}-\mathrm{V}$ conversion rate while maintaining the large collec- 
tive coupling constants [25]. Alternatively, this problem could be overcome by applying the external driving field to the electron spins on the Nitrogen atoms. It would increase the coherence time of the NVE if these spins are flipped on a time scale much faster than the flip-flop processes [26]. On the other hand, the dephasing time can be greatly enhanced by decoupling the electron spin from its local environment with a spin echo sequence. Using this technique, the dephasing time of the NVE reaches $3.7 \mu$ s at room temperature [25].

In summary, we have discussed how to simulate gauge field in a J-C lattice of NVE-TLR system, where the motion of polaritons (collective excitations) in NVEs is analogous to the motion of charged bosonic particles in a magnetic field. We have discussed the competition between the size effect and the gauge field on the ground state crossover, and also discussed the possibility of identifying signatures of the $\mathrm{HB}$ in the optical spectra of the superconducting resonators. With currently available techniques, we argue that our system lends itself as a well-suited quantum simulator for investigating phenomena encountered in condensed matter physics, and our study would be useful for the future spintronics technology.

We thanks Cong-jun $\mathrm{Wu}$ and Hong-hao $\mathrm{Tu}$ for valuable discussions. This work is supported by the NFRP of China under Grants 2011CBA00300, 2011CBA00301, No. 2011CB922102, No. 2011CB921200, No. 2011CBA00200, No. 2011CB921803, No. 2012CB921704, and by NNSF of China under Grants No. 10974225, No. 11004226, No. 11104326, No. 11105136, No. 11174035, No. 61073174, No. 61033001, and No. 61061130540, and Chinese PRF under Grant NO. 20110490829, as well as by the NRFME, Singapore (Grant No. WBS: R-710-000-008-271).
[1] J. Dalibard et al., Rev. Mod. Phys. 83, 1523 (2011).

[2] D. R. Hofstadter, Phys. Rev. B 14, 2239 (1976).

[3] R. Feynman, Int. J. Theor. Phys. 21, 467 (1982).

[4] N. R. Cooper et al., Phys. Rev. Lett. 87, 120405 (2001); R. Bhat et al., Phys. Rev. Lett. 96, 060405 (2006); M. Polini et al., Phys. Rev. Lett. 95, 010401 (2005); V. Schweikhard et al., Phys. Rev. Lett. 99, 030401 (2007); R. A. Williams et al., Phys. Rev. Lett. 104, 050404 (2010).

[5] A. Klein and D. Jaksch, Europhys. Lett. 85, 13001 (2009); M. Rosenkranz et al., Phys. Rev. A 81, 013607 (2010).

[6] A. S. Sørensen, E. Demler, and M. D. Lukin, Phys. Rev. Lett. 94, 086803 (2005).

[7] K. Osterloh et al., Phys. Rev. Lett. 95, 010403 (2005).

[8] E. J. Mueller, Phys. Rev. A 70, 041603(R) (2004).

[9] D. Jaksch and P. Zoller, New J. Phys. 5, 56 (2003).

[10] A. R. Kolovsky, Europhys. Lett. 93, 20003 (2011).

[11] J. Ruseckas et al., Phys. Rev. Lett. 95, 010404 (2005).

[12] G. Juzeliūnas and P. Öhberg, Phys. Rev. Lett. 93, 033602 (2004); G. Juzeliūnas et al., Phys. Rev. A 71, 053614 (2005); A. Jacob et al., New J. Phys. 10, 045022 (2008).

[13] J. Cho and M. S. Kim, Phys. Rev. Lett. 107, 260402 (2011).

[14] J. Koch et al., Phys. Rev. A 82, 043811 (2010); A. D. Greentree and A. M. Martin, Physics, 3, 85 (2010); A. Nunnenkamp, J. Koch, and S. M. Girvin, New J. Phys. 13, 095008 (2011).

[15] A. Bermudez, T. Schaetz, and D. Porras, Phys. Rev. Lett. 107, 150501 (2011).

[16] M.Hafezi, et al., Nat. Phys. 7, 907 (2011).

[17] R. O. Umucalilar and I. Carusotto, Phys. Rev. A 84, 043804 (2011).

[18] J. Cho, D. G. Angelakis, and S. Bose, Phys. Rev. Lett. 101, 246809 (2008).

[19] Y.-J. Lin et al., Nature (London) 462, 628 (2009); Y.-J. Lin et al., Phys. Rev. Lett. 102, 130401 (2009).

[20] M. Aidelsburger et al., Phys. Rev. Lett. 107, 255301 (2011); E. J. Mueller, Physics 4, 107 (2011).
[21] Y.-J. Lin et al., Nat. Phys. 7, 531 (2011).

[22] Y. Kubo et al., Phys. Rev. Lett. 105, 140502 (2010).

[23] D. I. Schuster et al., Phys. Rev. Lett. 105, 140501 (2010).

[24] R. Amsüss et al., Phys. Rev. Lett. 107, 060502 (2011).

[25] Y. Kubo et al., Phys. Rev. Lett. 107, 220501 (2011).

[26] D. Marcos et al., Phys. Rev. Lett. 105, 210501 (2010).

[27] W. L. Yang et al., Phys. Rev. A 83, 022302 (2011); Phys. Rev. A 84, 010301(R) (2011); Phys. Rev. A 85, 022324 (2012).

[28] A. Imamoğlu, Phys. Rev. Lett. 102, 083602 (2009); T. Duty, Physics 3, 80 (2010).

[29] Y. Makhlin, G. Schon, and A. Shnirman, Rev. Mod. Phys. 73, 357 (2001); A. Wallraff et al., Nature (London) 431, 162 (2004).

[30] T. Gaebel et al., Nat. Phys. 2, 408 (2006); L. Childress et al., Science 314, 281 (2006); M. V. Gurudev Dutt et al., Science 316, 1312 (2007); L. Jiang, et al., Science 326, 267 (2009); G. Balasubramanian et al., Nat. Mater. 8, 383 (2009); P. Neumann, et al., Science 329, 542 (2010); I Aharonovich et al., Rep. Prog. Phys. 74, 076501 (2011).

[31] M. Mariantoni et al., Nat. Phys. 7, 287 (2011); A. A. Houck et al., Nat. Phys. 8, 292 (2012).

[32] M. Neeley et al., Nature (London) 467, 570 (2010); L. DiCarlo et al., Nature (London) 467, 574 (2010).

[33] D. Underwood et al., arXiv:1203.5363.

[34] Using the calculation method of the Ref. [P. Neumann et al., Nat. Phys. 6, 249 (2010)], we find that the dipolarinteraction strength between distant $\mathrm{NV}$ centers of the same ensemble is of the order of a few $\mathrm{Hz}$ in the case of the densities of NV ensemble is $1 \times 10^{15} \mathrm{~cm}^{-3}$ or less.

[35] Y. Yu et al., Science 296, 889 (2002); J. M. Martinis et al., Phys. Rev. Lett. 89, 117901 (2002); A. Blais et al., Phys. Rev. Lett. 90, 127901 (2003); A. M. Zagoskin et al., Phys. Rev. Lett. 97, 077001 (2006).

[36] Y. Hu and L. Tian, Phys. Rev. Lett. 106, 257002 (2011).

[37] A. Kamal, J. Clarke, and M. H. Devoret, Nat. Phys. 7, 311 (2011).

[38] K. Hammerer, A. S. Sørensen, and E. S. Polzik, Rev. Mod. Phys. 82, 1041 (2010).

[39] G. Möller and N. Cooper, Phys. Rev. A 82, 063625 
(2010).

[40] J. M. Gambetta, A. A. Houck, and A. Blais, Phys. Rev. Lett. 106, 030502 (2011); J. Schreier et al., Phys. Rev. B 77, 180502 (2008); B. R. Johnson et al., Nat. Phys. 6, 663 (2010).

[41] Y. Kubo et al., Phys. Rev. A 85, 012333 (2012).

[42] C. Albrecht et al., Phys. Rev. Lett. 86, 147 (2001).

[43] Our scheme can also be applied to other spin degrees of freedom, such as N substitution (P1) centers [23], several defects in silicon carbide ( $\mathrm{SiC}$ ) [4]], electronic spins of quantum dot coupled to microwave superconducting resonator(SC) [45], and cold atoms coupled to SC [46].
[44] W. F. Koehl et al., Nature (London) 479, 84 (2011).

[45] M. R. Delbecq et al., Phys. Rev. Lett. 107, 256804 (2011).

[46] J. Verdú et al., Phys. Rev. Lett. 103, 043603 (2011).

[47] G. D. Fuchs et al., Science 326, 1520 (2009).

[48] X. Zhu et al., Nature (London) 478, 221 (2011).

[49] P. Neumann et al., Science 320, 1326 (2008).

[50] J. Harrison et al., Diam. Relat. Mater. 15, 586 (2006).

[51] P. L. Stanwix, et al., Phys. Rev. B 82, 201201 (2010).

[52] G. Balasubramanian et al., Nat. Mater. 8, 383 (2009).

[53] Z.-R. Zhong et al., arXiv1110.4331. 


\section{Supplementary Material}

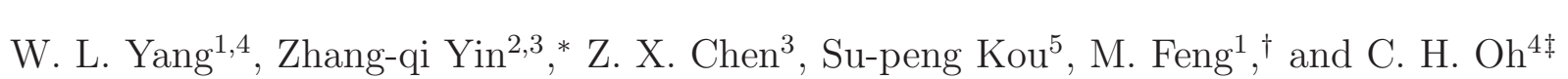

${ }^{1}$ State Key Laboratory of Magnetic Resonance and Atomic and Molecular Physics, Wuhan Institute of Physics and Mathematics, Chinese Academy of Sciences, and Wuhan National Laboratory for Optoelectronics, Wuhan 430071, China

${ }^{2}$ Key Laboratory of Quantum Information, University of Science and Technology of China, Chinese Academy of Sciences, Hefei 230026, China

${ }^{3}$ Center for Quantum Information, IIIS, Tsinghua University, Beijing, China

${ }^{4}$ Centre for Quantum Technologies, National University of Singapore, Singapore 117543, Singapore and ${ }^{5}$ Department of Physics, Beijing Normal University, Beijing 100875, China

*Electronic address: yinzhangqi@gmail.com

$\dagger$ Electronic address: mangfeng@wipm.ac.cn

${ }^{\ddagger}$ Electronic address: phyohch@nus.edu.sg 


\section{HAMILTONIAN FOR THE SINGLE-SITE NVE-TLR}

We suppose that each NVE couples to a two-mode TLR in each site. By applying an external resonant driving microwave on the NVE, the Hamiltonian of the $(p, q)$-th site reads

$$
\begin{aligned}
H_{p, q}= & \sum_{c=a, b} \omega_{c} c_{p, q}^{\dagger} c_{p, q}+\sum_{j=1}^{N_{p, q}} \omega_{e}|e\rangle_{p, q p, q}^{j j}\langle e|+\left[\sum _ { j = 1 } ^ { N _ { p , q } } \left(\frac{\Omega_{p, q} e^{i \theta_{p, q}}}{2} e^{-i \omega_{e} t}|e\rangle_{p, q p, q}^{j j}\langle g|\right.\right. \\
& \left.\left.+\sum_{c=a, b} g_{p, q}^{c} c_{p, q}|e\rangle_{p, q p, q}^{j}\langle g|\right)+ \text { H.C. }\right],
\end{aligned}
$$

where $\Omega_{p, q}$ is the Rabi frequency of the driving microwave, and $\theta_{p, q}$ is the relative phase. In the rotating frame regarding $H_{0}=\sum_{c=a, b} \omega_{e} c_{p, q}^{\dagger} c_{p, q}+\omega_{e} \sum_{j=1}^{N_{p, q}}|e\rangle_{p, q p, q}^{j}\langle e|$, we get the Hamiltonian as

$$
H_{p, q}=\sum_{c=a, b}\left(\omega_{c}-\omega_{e}\right) c_{p, q}^{\dagger} c_{p, q}+\left[\sum_{j=1}^{N_{p, q}}\left(\frac{\Omega_{p, q} e^{i \theta_{p, q}}}{2}|e\rangle_{p, q p, q}^{j j}\left\langle g\left|+\sum_{c=a, b} g_{p, q}^{c} c_{p, q}\right| e\right\rangle_{p, q p, q}^{j}\langle g|\right)+\text { H.C. }\right] .
$$

Here we define $\Delta_{c}=\omega_{c}-\omega_{e}$ for $c=a, b$.

Under the strong driving case $\Omega_{p, q} \gg g_{p, q}^{c}$, we can define the new energy levels as $|+\rangle_{p, q}=$ $\left(e^{i \theta_{p, q}}|e\rangle_{p, q}+|g\rangle_{p, q}\right) / \sqrt{2}$ and $|-\rangle_{p, q}=\left(-e^{i \theta_{p, q}}|e\rangle_{p, q}+|g\rangle_{p, q}\right) / \sqrt{2}$. The Hamiltonian of the system under the new basis is

$$
\begin{aligned}
H_{p, q}= & \sum_{c=a, b} \Delta_{c} c_{p, q}^{\dagger} c_{p, q}+\sum_{j=1}^{N_{p, q}}\left(\frac{\left|\Omega_{p, q}\right|}{2}\left(|+\rangle_{p, q p, q}^{j}\langle+|-|-\rangle_{p, q p, q}^{j}\langle-|\right)\right. \\
& +\frac{1}{2} \sum_{c=a, b} g_{p, q}^{c} c_{p, q} e^{-i \theta_{p, q}}\left(|+\rangle_{p, q}^{j}-|-\rangle_{p, q}^{j}\right)\left({ }_{p, q}^{j}\langle+|+{ }_{p, q}^{j}\langle-|\right) \\
& \left.+\frac{1}{2} \sum_{c=a, b} g_{p, q}^{c} c_{p, q}^{\dagger} e^{i \theta_{p, q}}\left(|+\rangle_{p, q}^{j}+|-\rangle_{p, q}^{j}\right){ }_{p, q}^{j}\langle+|--_{p, q}^{j}\langle-|\right) .
\end{aligned}
$$

Let's neglect the energy shift induced by the cavity mode, which is much smaller than the energy shift induced by the driving laser. Then we obtain

$$
\begin{aligned}
H_{p, q}= & \sum_{c=a, b} \Delta_{c} c_{p, q}^{\dagger} c_{p, q}+\sum_{j=1}^{N_{p, q}}\left(\frac{\left|\Omega_{p, q}\right|}{2}\left(|+\rangle_{p, q p, q}^{j}\langle+|-|-\rangle_{p, q p, q}^{j}\langle-|\right)\right. \\
& +\frac{1}{2} \sum_{j=1}^{N_{p, q}} \sum_{c=a, b}\left[g_{p, q}^{c} c_{p, q} e^{-i \theta_{p, q}}\left(|+\rangle_{p, q p, q}^{j}\langle-|-|-\rangle_{p, q p, q}^{j}\langle+|\right)+\text { H.C. }\right] .
\end{aligned}
$$

Suppose $\Delta_{a} \simeq\left|\Omega_{p, q}\right|, \Delta_{b} \simeq-\left|\Omega_{p, q}\right|$, and $\Delta_{c}, \Omega_{p, q} \gg \sqrt{N_{p, q}} g_{p, q}^{c}$, we can obtain the effective 
Hamiltonian under the rotating wave approximation,

$$
\begin{aligned}
H_{p, q}^{e f f}= & \delta_{p, q}^{a} a_{p, q}^{\dagger} a_{p, q}+\delta_{p, q}^{b} b_{p, q}^{\dagger} b_{p, q}+\frac{1}{2} \sum_{j=1}^{N_{p, q}}\left[g_{p, q}^{a} a_{p, q} e^{-i \theta_{p, q}}|+\rangle_{p, q p, q}^{j j}\langle-|\right. \\
& \left.-g_{p, q}^{b} b_{p, q}^{\dagger} e^{-i \theta_{p, q}}|+\rangle_{p, q p, q}^{j}\langle-|+\text { H.C. }\right],
\end{aligned}
$$

where $\delta_{p, q}^{a}=\Delta_{a}-\Omega_{p, q}$ and $\delta_{p, q}^{b}=\Delta_{b}+\Omega_{p, q}$. Let's define $\tilde{S}_{p, q}^{+}=\sum_{j=1}^{N_{p, q}}|+\rangle_{p, q}^{j}\langle-|$ and $\tilde{S}_{p, q}^{-}=\sum_{j=1}^{N_{p, q}}|-\rangle_{p, q}^{j}\langle+|$ for $(p, q)$-th site. We denote the Hamiltonian of the coupling between mode $a$ and NVE by $H_{p, q}^{a}=\delta_{p, q}^{a} a_{p, q}^{\dagger} a_{p, q}+\left(\frac{1}{2} g_{p, q}^{a} e^{-i \theta_{p, q}} a_{p, q} \tilde{S}_{p, q}^{+}+\right.$H.C. $)$. On the other hand, the effective Hamiltonian describing the coupling between mode $b$ and NVE is given by $H_{p, q}^{b}=\delta_{p, q}^{b} b_{p, q}^{\dagger} b_{p, q}-\left(\frac{1}{2} g_{p, q}^{b} e^{-i \theta_{p, q}} b_{p, q}^{\dagger} \tilde{S}_{p, q}^{+}+\right.$H.C. $)$.

In the rotating frame regarding $H_{0}=\delta_{p, q}^{a} a_{p, q}^{\dagger} a_{p, q}+\delta_{p, q}^{b} b_{p, q}^{\dagger} b_{p, q}$, we rewrite Eq.(4) as

$$
H_{p, q}^{e f f}=\frac{1}{2} g_{p, q}^{a} e^{-i\left(\theta_{p, q}+\delta_{p, q}^{a} t\right)} a_{p, q} \tilde{S}_{p, q}^{+}-\frac{1}{2} g_{p, q}^{b} e^{-i\left(\theta_{p, q}-\delta_{p, q}^{b} t\right)} b_{p, q} \tilde{S}_{p, q}^{+}+\text {H.C.. }
$$

\section{EFFECTIVE HAMILTONIAN FOR COUPLED CAVITY SITES}

The coupling between $(p, q)$-th site and the nearest TLR sites can be modeled by following Hamiltonian.

$$
H_{T}=-\sum_{p, q=1}^{N} \hbar\left(T_{a} a_{p, q+1}^{\dagger} a_{p, q}+T_{a} a_{p, q}^{\dagger} a_{p, q+1}+T_{b} b_{p+1, q} b_{p, q}^{\dagger}+T_{b} b_{p, q} b_{p+1, q}^{\dagger}\right)
$$

We diagonalize the Hamiltonian (6) with the Fourier transformation $a_{k_{q}, p}=$ $\frac{1}{\sqrt{N}} \sum_{q=1}^{N} e^{-i 2 \pi k_{q} q / N} a_{p, q}$ and $b_{k_{p}, q}=\frac{1}{\sqrt{N}} \sum_{p=1}^{N} e^{-i 2 \pi k_{p} p / N} b_{p, q}$,

$$
H_{T}^{\prime}=-\sum_{k_{p}=2 \pi / N}^{2 \pi} \sum_{q=1}^{N} T_{k_{p}} b_{k_{p}, q}^{\dagger} b_{k_{p}, q}-\sum_{k_{p}=2 \pi / N}^{2 \pi} \sum_{p=1}^{N} T_{k_{p}} a_{k_{p}, p}^{\dagger} a_{k_{p}, p},
$$

where $T_{k_{p}}=2 T_{b} \cos \left(2 \pi k_{p} / N\right)$ and $T_{k_{q}}=2 T_{a} \cos \left(2 \pi k_{q} / N\right)$. We define $F_{p, k_{p}}=e^{-i 2 \pi k_{p} p / N}$ and $F_{q, k_{q}}=e^{-i 2 \pi k_{q} q / N}$.

It is found that the coupling between vertical sites and between horizonal sites can be calculated independently. The effective Hamiltonian of the system for the vertical line $q$ is

$$
H_{v}=-\sum_{p=1}^{N}\left(\frac{1}{2} g_{p, q}^{b} e^{-i\left(\theta_{p, q}-\delta_{p, q}^{b} t\right)} b_{p, q} \tilde{S}_{p, q}^{-}+\text {H.C. }\right)-\sum_{k_{p}=2 \pi / N}^{2 \pi} T_{k_{p}} b_{k_{p}, q}^{\dagger} b_{k_{p}, q} .
$$


For simplicity, we assume $\delta_{p, q}^{a}=\delta_{p, q}^{b}=\delta, g_{p, q}^{a}=g_{p, q}^{b}=g$, and $T_{p, q}=T$. With the transformation $b_{k_{p}, q}=\sum_{p=1}^{N} F_{k_{p}, p} b_{p, q}$, we get

$$
H_{v}=-\sum_{p=1}^{N} \sum_{k_{p}=2 \pi / N}^{2 \pi}\left(\frac{1}{2 \sqrt{N}} g F_{k_{p}, p} b_{k_{p}, q} \tilde{S}_{p, q}^{-} e^{-i \theta_{p, q}} e^{i(T+\delta) t}+\text { H.C. }\right) \text {. }
$$

In the limit of $\delta \gg T, g$, we can adiabatically eliminate the cavity modes $b_{k_{p}, q}$ and get

$$
H_{v}^{e f f}=\sum_{p, p^{\prime}}^{N} \frac{g^{2}}{4} e^{i\left(\theta_{p^{\prime}, q}-\theta_{p, q}\right)} \tilde{S}_{p^{\prime}, q}^{+} \tilde{S}_{p, q}^{-} \sum_{k_{p}=2 \pi / N}^{2 \pi} \frac{F_{k_{p}^{\prime}} F_{k_{p}}^{*}}{N} \frac{1}{\delta+T_{k_{p}}} .
$$

In the limit of $\delta \gg T$, we get $1 /\left(\delta+T_{k_{p}}\right) \simeq \frac{1}{\delta}-\frac{T_{k_{p}}}{\delta^{2}}, \sum_{k_{p}} F_{k_{p}, p} F_{k_{p}, p^{\prime}}^{*}=N \delta_{p, p^{\prime}}$, and $\sum_{k_{p}} F_{k_{p}, p} F_{k_{p}, p^{\prime}}^{*} T_{k_{p}}=T\left(\delta_{p, p^{\prime}+1}+\delta_{p+1, p^{\prime}}\right)$. Neglecting the Stark shift terms, we get the effective Hamiltonian as

$$
H_{v}^{e f f}=\sum_{p, p^{\prime}}^{N} \frac{T g^{2}}{4 \delta^{2}} e^{i\left(\theta_{p+1, q}-\theta_{p, q}\right)} \tilde{S}_{p+1, q}^{+} \tilde{S}_{p, q}^{-}+\text {H.C. }
$$

Similarly, we can calculate the effective Hamiltonian for the horizontal line $p$ by adiabatically eliminating the cavity modes as the following form,

$$
H_{h}^{e f f}=\sum_{q, q^{\prime}}^{N} \frac{T g^{2}}{4 \delta^{2}} e^{i\left(\theta_{p, q}-\theta_{p, q+1}\right)} \tilde{S}_{p, q+1}^{+} \tilde{S}_{p, q}^{-}+\text {H.C. }
$$

Combining Eqs. (8) and (11), we obtain the effective Hamiltonian for the whole system as

$$
\begin{aligned}
H_{\mathrm{eff}}= & -J^{\prime}\left(\sum_{p, q} e^{i\left(\theta_{p, q}-\theta_{p+1, q}\right)} \tilde{S}_{p, q}^{+} \tilde{S}_{p+1, q}^{-}\right. \\
& \left.+e^{i\left(\theta_{p, q+1}-\theta_{p, q}\right)} \tilde{S}_{p, q}^{+} \tilde{S}_{p, q+1}^{-}+\text {H.C. }\right),
\end{aligned}
$$

where $J^{\prime}=T(g / 2 \delta)^{2}$.

\section{THE GROUND STATE NEAR LEVEL CROSSING}

We find that the ground state level crossing can be observed in the system as small as $5 \times 5$ lattices. As shown in Fig, the critical value of gauge field is $\alpha_{0}=0.333 \simeq 1 / 3$.

The numerical result of the ground state near the critical gauge field $\alpha_{0}$ for the $5 \times 5$ 

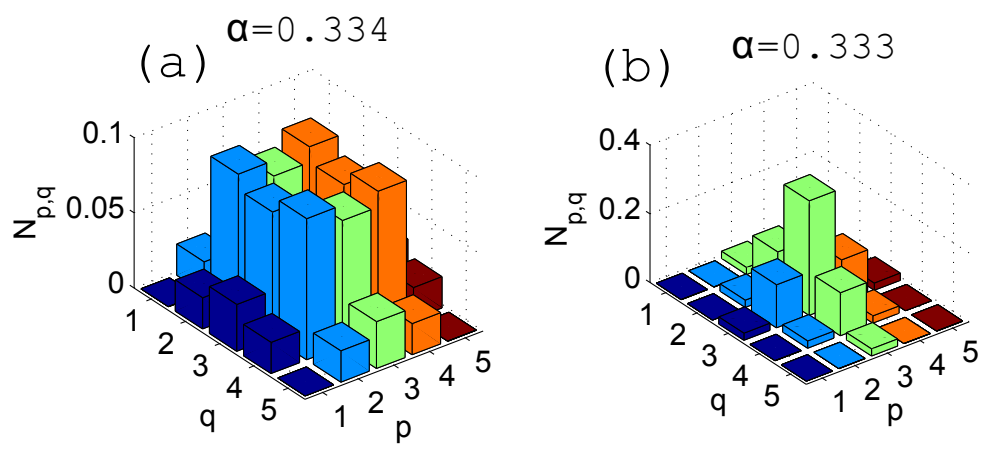

FIG. 1: (Color online) The spatial distribution of the NVE polaritons for a $5 \times 5$ lattice under different values of $\alpha$. (a) $\alpha=0.334$. (b) $\alpha=0.333$.

lattice system is shown as follows,

$$
\begin{aligned}
\Phi(0.333)= & 0|1\rangle_{1,1}+(0.0003-0.0002 i)|1\rangle_{1,2}+(0.144-0.0009 i)|1\rangle_{1,3} \\
& +(0.0003+0.0002 i)|1\rangle_{1,4}+0|1\rangle_{1,5} \\
& +(0.0003+0.0002 i)|1\rangle_{2,1}+0.1448|1\rangle_{2,2}+(0.3536-0.0011 i)|1\rangle_{2,3} \\
& +(0.1448-0.0009 i)|1\rangle_{2,4}+(0.0003-0.0002 i)|1\rangle_{2,5} \\
& +(0.1440+0.0009 i)|1\rangle_{3,1}+(0.3536+0.0011 i)|1\rangle_{3,2}+0.5772|1\rangle_{3,3} \\
& +(0.3536-0.0011 i)|1\rangle_{3,4}+(0.144+0.0009 i)|1\rangle_{3,5} \\
& +(0.0003-0.0002 i)|1\rangle_{4,1}+(0.1448+0.0009 i)|1\rangle_{4,2} \\
& +(0.3536+0.0011 i)|1\rangle_{4,3}+0.1448|1\rangle_{4,4}+(0.0003+0.0002 i)|1\rangle_{4,5} \\
& +0|1\rangle_{5,1}+(0.0003+0.0002 i)|1\rangle_{5,2}+(0.1440+0.0009 i)|1\rangle_{5,3} \\
& +(0.0003-0.0002 i)|1\rangle_{5,4}+0|1\rangle_{5,5} .
\end{aligned}
$$




$$
\begin{aligned}
\Phi(0.334)= & -0.0004|1\rangle_{1,1}+(0.1251-0.00713 i)|1\rangle_{1,2}+(0.0022-0.1763 i)|1\rangle_{1,3} \\
& +(-0.1232+0.00745 i)|1\rangle_{1,4}+0.0004|1\rangle_{1,5} \\
& +(0.1251+0.0713 i)|1\rangle_{2,1}+0.3065|1\rangle_{2,2}+(0.0018-0.2890 i)|1\rangle_{2,3} \\
& +(0.3064-0.0039 i)|1\rangle_{2,4}+(0.1260-0.0698 i)|1\rangle_{2,5} \\
& +(0.0022+0.1763 i)|1\rangle_{3,1}+(0.0018+0.2890 i)|1\rangle_{3,2}+0|1\rangle_{3,3} \\
& +(-0.0018+0.2890 i)|1\rangle_{3,4}+(-0.0022+0.1763 i)|1\rangle_{3,5} \\
& +(-0.1232+0.0745 i)|1\rangle_{4,1}+(-0.3064+0.0039 i)|1\rangle_{4,2} \\
& +(-0.0018-0.2890 i)|1\rangle_{4,3}+0.3065|1\rangle_{4,4}+(0.1242+0.00729 i)|1\rangle_{4,5} \\
& +0.0004|1\rangle_{5,1}+(-0.1260+0.0698 i)|1\rangle_{5,2}+(-0.0022+0.1763 i)|1\rangle_{5,3} \\
& +(0.1242-0.0729 i)|1\rangle_{5,4}-0.0004|1\rangle_{5,5} .
\end{aligned}
$$

Here $|\Psi(\alpha)\rangle$ denotes the ground state at the field $\alpha$, and $|1\rangle_{p, q}$ denotes the Fock state $|1\rangle$ of the polariton at the lattice site $p, q$. It is easy to verify that $\langle\Phi(0.333) \mid \Phi(0.334)\rangle=$ $1.58 \times 10^{-5} \sim 0$. Therefore, there is level crossing near the critical gauge field $\alpha_{0}=0.333$.

In general, we can define the ground state fidelity for the system $F(\alpha)=\langle\Phi(\alpha) \mid \Phi(\alpha+\delta)\rangle$, with $\delta \rightarrow 0$. For those $F(\alpha)$ approach zero, the level crossing occurs. We have numerically calculated the $F(\alpha)$ and identified the level crossing points for different sizes of lattices. For example, for $5 \times 5$ lattice, the level crossing point is near $1 / 3$. For $6 \times 6$ lattice, the level crossing points are near $2 / 7,3 / 8,2 / 5$ and $1 / 2$. The critical points are fitted very well with rational number $p / q$ for different lattices, and the first crossing point $\alpha_{0}$ is just $2 /(L+1)$ for the $L \times L$ systems $(L>4)$. To qualitatively learn the nature of the first level crossing at $\alpha_{0}=2 /(L+1)$, we estimate $\alpha_{0}$ by perturbation approach.

First we consider a zero magnetic field case, $\alpha=0$. Now the effective model in Eq.(12) is reduced to a continuum form as

$$
H_{B} \rightarrow \frac{\hat{k}_{p}^{2}}{2 m}+\frac{\hat{k}_{q}^{2}}{2 m},
$$

where $m=\frac{1}{2 J}$. Considering the boundary condition,

$$
\begin{gathered}
\Psi(0,0)=0, \Psi\left(L_{p}, 0\right)=0, \\
\Psi\left(0, L_{q}\right)=0, \Psi\left(L_{p}, L_{q}\right)=0,
\end{gathered}
$$


where $L_{p}\left(L_{q}\right)$ is the number sites along $\mathrm{p}(\mathrm{q})$ direction. We have the wave-function as

$$
\Psi_{(p, q)}=\frac{2}{\sqrt{L_{p} L_{q}}} \sin \left(\frac{\pi p}{L_{q}}\right) \sin \left(\frac{\pi q}{L_{p}}\right)
$$

and the energy eigenvalues as

$$
E_{(p, q)}=\frac{1}{2 m}\left[\left(\frac{\pi p}{L_{p}}\right)^{2}+\left(\frac{\pi q}{L_{q}}\right)^{2}\right]
$$

where $p=1,2,3, . . L_{p}$ and $q=1,2,3, . . L_{q}$. It means that the ground state for the $\alpha \rightarrow 0$ case is labeled by $p=1, q=1$ with the energy

$$
E_{(1,1)}=\frac{1}{2 m}\left[\left(\frac{\pi p}{L_{p}}\right)^{2}+\left(\frac{\pi q}{L_{q}}\right)^{2}\right]
$$

The first excited state is labeled by $p=2, q=1$ or $p=1, q=2$ with the energy

$$
\min \left(E_{(2,1)}, E_{(1,2)}\right)=\min \left(\frac{1}{2 m}\left[\left(\frac{2 \pi p}{L_{p}}\right)^{2}+\left(\frac{\pi q}{L_{q}}\right)^{2}\right], \frac{1}{2 m}\left[\left(\frac{\pi p}{L_{p}}\right)^{2}+\left(\frac{2 \pi q}{L_{q}}\right)^{2}\right]\right) .
$$

Secondly we add a tiny magnetic field $\alpha$. The energy spectrum of the system changes little. So we may use the perturbation theory to deal with this case. Here we choose the Landau gauge, $\vec{A}=(B y, 0)$, where the gauge field strength $B=2 \pi \alpha$. In the following part we set $L_{x}=L_{x}=L$.

Now the model in Eq.(12) in a continuum form turns into

$$
H_{B}^{\prime} \rightarrow \frac{\left(\hat{k}_{p}-B q\right)^{2}}{2 m}+\frac{\hat{k}_{q}^{2}}{2 m}
$$

In the perturbation theory, we use the plane waves of non-magnetic case $\Psi_{(p, q)}$ to describe above model. Then for the lowest eigenstate $\Psi_{(1,1)}$, and the excited state $\Psi_{(2,1)}$, we have the corresponding perturbation energies as

$$
E_{(1,1)}^{\prime}=\frac{1}{2 m}\left[\left(\frac{\pi}{L}\right)^{2}\right]+\frac{1}{2 m}\left[\left(\frac{\pi}{L}\right)^{2}\right]+\frac{1}{L^{2}} \frac{1}{2 m} \sum_{q=1}^{L} 2 \frac{\pi}{L}(-2 \pi \alpha q)+\alpha^{2} \text { term, }
$$

and

$$
E_{(2,1)}^{\prime}=\frac{1}{2 m}\left[\left(\frac{\pi}{L}\right)^{2}\right]+\frac{1}{2 m}\left[\left(\frac{2 \pi}{L}\right)^{2}\right]+\frac{1}{L^{2}} \frac{1}{2 m} \sum_{q=1}^{L} 2 \frac{2 \pi}{L}(-2 \pi \alpha q)+\alpha^{2} \text { term }
$$

where the $\alpha^{2}$ terms can be neglected when $\alpha \ll 1$. So there may exist level crossing between $\Psi_{(1,1)}$ and $\Psi_{(2,1)}$ when

$$
E_{(1,1)}^{\prime}=E_{(2,1)}^{\prime}
$$




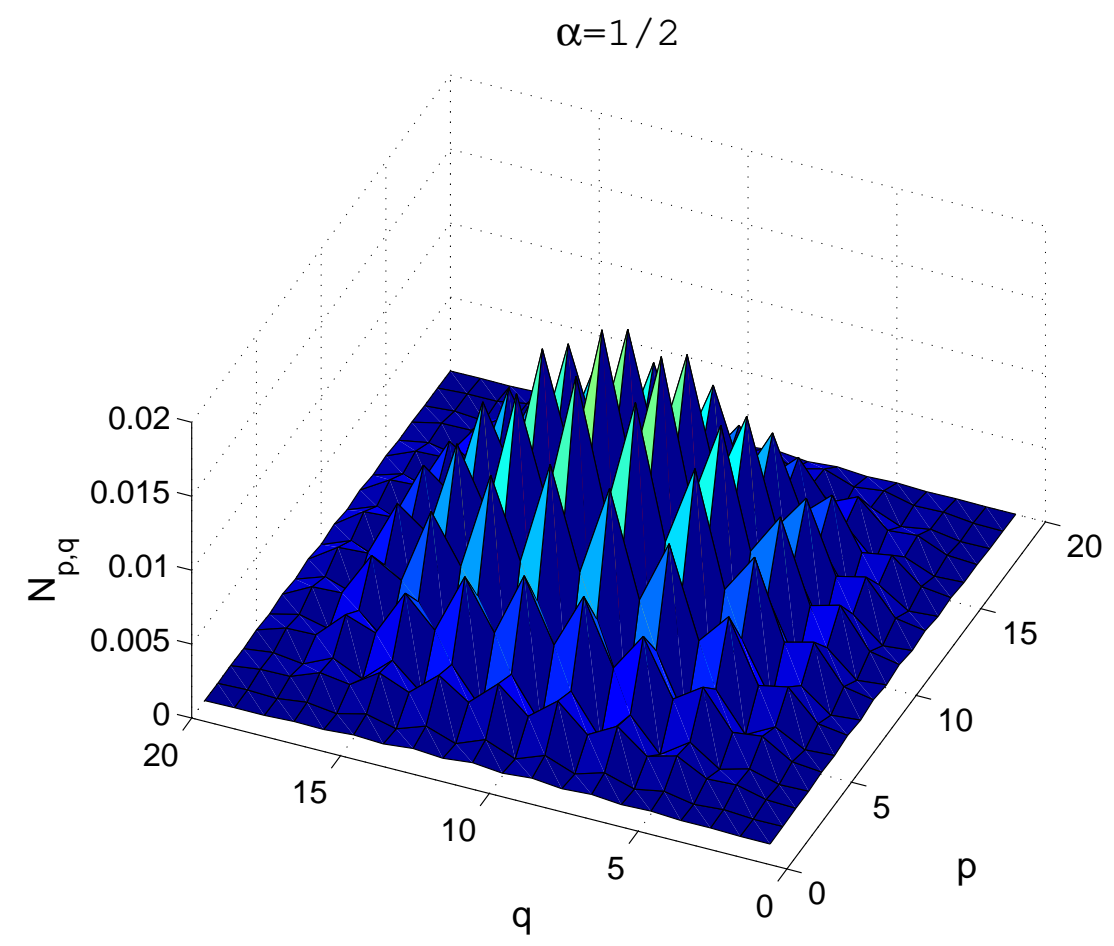

FIG. 2: The total fermion density of $\pi$-flux case on $20 \times 20$ lattice.

that is

$$
\frac{1}{2 m}\left[\left(\frac{2 \pi}{L}\right)^{2}\right]-\frac{1}{2 m}\left[\left(\frac{\pi}{L}\right)^{2}\right]=\frac{1}{L^{2}} \frac{1}{2 m} \sum_{q=1}^{L} 2 \frac{2 \pi}{L}\left(2 \pi \alpha_{c} q\right)-\frac{1}{L^{2}} \frac{1}{2 m} \sum_{q=1}^{L} 2 \frac{\pi}{L}\left(2 \pi \alpha_{c} q\right)
$$

for tiny $\alpha$. That is

$$
\frac{3}{2 m}\left(\frac{\pi}{L}\right)^{2}=\frac{1}{L^{2}} \frac{1}{2 m} \sum_{q=1}^{L} 2 \frac{\pi}{L}\left(2 \pi \alpha_{c} q\right)
$$

or

$$
\alpha_{c}=\frac{3}{2} \frac{1}{(L+1)}
$$

with $\sum_{q=1}^{L} q=\frac{L(L+1)}{2}$. Therefore we estimate the first level crossing at $\alpha_{0}=1.5 /(L+1)$ by perturbation approach, which is qualitatively same as the numerical result $\alpha_{0}=2 /(L+1)$.

\section{INTERFERENCE PATTERN FOR $\alpha=p / q$}

From the ground state fidelity we have found that all the level crossing points are near certain rational points $\alpha=p / q$, at which the wave-functions show regular oscillations. Fig. 2 illustrates the particle density of $\alpha=\frac{1}{2}$ case on $20 \times 20$ lattice. After Fourier 


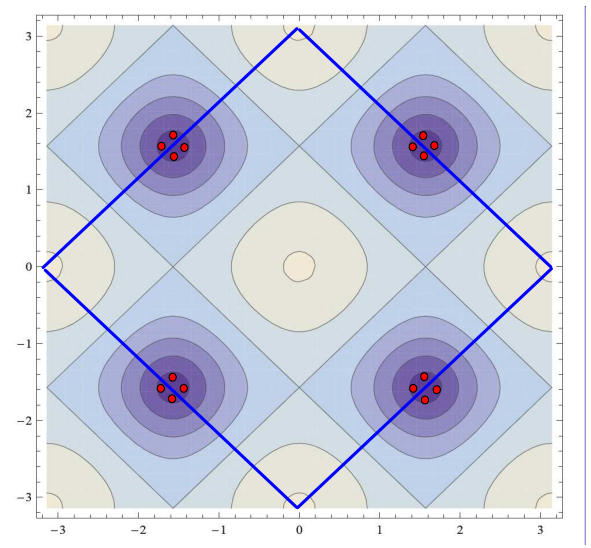

FIG. 3: The illustration of interference pattern of $\pi$-flux case on $20 \times 20$ lattice in momentum space. The blue lines are the boundary of reduced BZ. The 8 resonant points is

$$
\left(k_{p}, k_{q}\right)=\left(\frac{\pi}{2} \pm \frac{\pi}{20}, \pm \frac{\pi}{2} \pm \frac{\pi}{20}\right) .
$$

transformation, we find that such regular oscillations come from the coherent interference between the peculiar points in momentum space. Thus for the ground state of $\alpha=\frac{1}{2}$ case on an $L \times L$ lattice with open boundary condition, the particle density can be transformed into 8 resonant points inside the reduced Brillouin zone (BZ) of momentum space as

$$
\left(k_{p}, k_{q}\right)=\left(\frac{\pi}{2} \pm \frac{\pi}{L}, \pm \frac{\pi}{2} \pm \frac{\pi}{L}\right)
$$

around the nodal points $\left(k_{p}, k_{q}\right)=\left(\frac{\pi}{2}, \pm \frac{\pi}{2}\right)$. Let's explain this result. For an infinite system, the case of $\alpha=\frac{1}{2}$ is just a $\pi$-flux model, of which there are two nodal points in the reduced BZ as $\left(\frac{\pi}{2}, \pm \frac{\pi}{2}\right)$. To transform the wave-function of the system with finite size, we can consider the $L \times L$ lattice with open boundary condition as an infinite system with periodic $L \times L$ super-cells, of which the wave-functions are fixed to be zero at the interfaces between two $L \times L$ super-cells. Thus, modulated by $L \times L$ super-cells, each nodal points split to 4 points. As a result, we can read out the information of a model with the periodic condition in thermodynamic limit in this small and open boundary system. As shown in Fig. 3, for a $20 \times 20$ lattice, the 8 resonant points in the reduced BZ zone are already very close to the 2 nodal points for an infinite system. 